REVIEW ARTICLE

\title{
A Review on Interventions against COVID-19
}

\author{
Sintayehu Tsegaye Tseha ${ }^{1,2^{*}}$
}

${ }^{1}$ Infection Biology PhD Candidate, Department of Microbial, Cellular and Molecular Biology, Addis Ababa University, Ethiopia

${ }^{2} \mathrm{MSc}$ in Biomedical Sciences, Lecturer of Biomedical Sciences, Department of Biology, Arba Minch University, Ethiopia

*Corresponding author: Sintayehu Tsegaye Tseha, Infection Biology PhD Candidate, Department of Microbial, Cellular and Molecular Biology, Addis Ababa University, Ethiopia; MSc in Biomedical Sciences, Lecturer of Biomedical Sciences, Department of Biology, Arba Minch University, Ethiopia

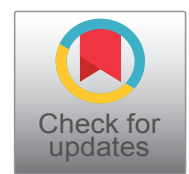

\begin{abstract}
COVID-19 remains the main public health problem in the world. The major objective of this review is to discuss interventions that have been employed against COVID-19. The preventive and control measures that have been employed against COVID-19 include: staying at home, social and physical distancing, quarantine, washing hand with sanitizer, using face mask, immunization, timely diagnosis and treatment of the disease. In spite of its high false negative rate, real time-PCR remains the gold standard for diagnosis of COVID-19. Antiviral drugs that have been approved for treatment of COVID-19 include remedsvier, monoclonal antibodies and convalescent plasma therapy. The vaccines that were authorized and approved for COVID-19 have shown positive outcomes. For example, a vaccine efficacy studies that done in Israel on efficacies of Moderna and Pfizer-BioNTech vaccines showed that the two vaccines reduce SARS-CoV-2 infection rate by more than $90 \%$. However, it is still possible for fully vaccinated people to contract and spread SARS-CoV-2 because of the high mutation rate of SARS-CoV-2. As the SARS-CoV-2 is constantly changing by random mutation, modification of the current vaccines is required to develop effective vaccines against all variants of the virus. In addition, as the available COVID-19 diagnosis methods have considerable limitations, a novel diagnostic approach that may overcome the limitations has to be designed.
\end{abstract}

\section{Keywords}

COVID-19, SARS-CoV-2, Interventions

\section{Introduction}

Coronavirus disease 2019 (COVID-19) remains the main public health problem in the world. According to World Health Organization (WHO), up to $13^{\text {th }}$
September 2021, the number of confirmed COVID-19 cases and deaths due to the disease reached more than $224,372,380$ and $4,625,006$ deaths, respectively, in the world [1]. This new disease was first identified in Wuhan city, China, in December 2019 and the disease was named COVID-19 on February 11, 2020 [2]. The disease was declared pandemic by WHO on $11^{\text {th }}$ March 2020 [3]. The worldwide spread of SARSCoV-2 has caused widespread social and economic disruption and overwhelmed health systems. The major objective of this review is to discuss interventions that have been used against COVID-19.

\section{Virology}

\section{Structure of SARS-CoV-2}

SARS-CoV-2 is an enveloped virus with single stranded positive sense RNA genome. The virus is characterized by crown-like spikes on the outer surface. SARS-CoV-2 is the largest known RNA virus which has a genome size ranging from 26-32 kilobases [4]. The virion of coronaviruses (CoVs) has diameters of approximately $125 \mathrm{~nm}[5]$ and has helically symmetrical nucleocapsids [6].

Like other coronaviruses, SARS-CoV-2 contains at least ten open reading frames (ORFs) [6]. The first ORFs (ORF1a/b), about two-thirds of viral RNA, are translated into two large polyproteins (pp). The two polyproteins (pp1a and $\mathrm{pp} 1 \mathrm{~b}$ ) are processed into 16 non-structural proteins (nsp) in sever acute respiratory syndrome associated coronavirus (SARS-CoV) and Middle East

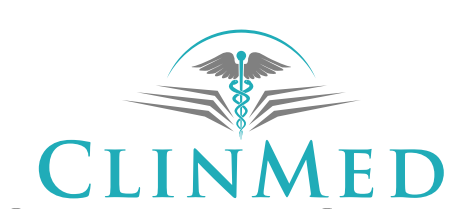

INTERNATIONAL LIBRARY
Citation: Tseha ST (2021) A Review on Interventions against COVID-19. Int J Virol AIDS 8:076. doi. org/10.23937/2469-567X/1510076

Accepted: September 22, 2021: Published: September 24, 2021

Copyright: (C) 2021 Tseha ST. This is an open-access article distributed under the terms of the Creative Commons Attribution License, which permits unrestricted use, distribution, and reproduction in any medium, provided the original author and source are credited. 
respiratory syndrome related coronavirus (MERS-CoV) that forms the viral replicase transcriptase complex [6]. The other ORFs of the viral genome encode five major structural proteins, which include: spike protein $(S)$, envelop protein $(E)$, membrane protein $(M)$, nucleocapsid (N), and hemagglutinin esterase (HE) [7].

The spike glycoprotein is located on the outer part of the virus, which protrudes in the viral surface and binds with host cell receptor which is called angiotensin converting enzyme-2 (ACE-2) [8]. The ACE-2 is highly expressed in lower respiratory tract of lungs, upper esophagus, epithelial cells, myocardial cells, and kidney cells [9]. The spike glycoprotein has two components (sub-units), namely S1 and S2. The function of S1 is to bind with host cell receptor with its receptor binding domain (RBD) while the S2 sub-unit mediates the fusion of the virus into host cell [10].

The nucleocapsid covers the genome of the virus, which is involved in the viral replication cycle [11]. Membrane protein (M) determines the shape of the virus. Envelop protein (E) facilitates assembly and release of the virus [12]. Haemagglutinin-esterase has two roles: it acts as haemagglutinin that has host receptor binding activity and has acetyl-esterase activity, which has receptor hydrolysis role [13]. The receptor binding and receptor hydrolysis roles of hemagglutinin esterase enhance the spike protein mediated cell entry of SARSCoV-2 virus into host cell [14].

\section{Taxonomy of SARS-CoV-2}

Coronaviruses belong to the family Coronaviridae. Coronaviridae contains two subfamilies: Coronavirinae and Torovirinae. Sub-family Coronavirinae is further classified into four genera; which includes genus Alpha coronavirus, genus Beta coronavirus, genus gamma coronavirus and genus delta coronavirus [15]. Alpha and ßeta-coronaviruses are able to infect mammals [15], whereas delta and gamma coronaviruses infect birds [16]. The Beta genus is further divided into four subgenera, namely Nobecovirus, Merbecovirus, Sarbecovirus, and Embecovirus [17].

Before the discovery of SARS-CoV2, six species of human coronaviruses ( $\mathrm{HCoV}$ ) have been identified to cause human disease, namely, HCoV-229E, HCoV-HKU1, HCoV-NL63, HCOVOC-43, MERS-CoV and SARS-CoV. MERS-CoV and SARS-CoV usually bring about severe and fatal respiratory diseases [18]. Whereas, HCoVHKU1, HCoV-OC43, HCoV-229E and HCoV-NL63 cause mild illness like common cold [19].

SARS-CoV-2 is the $7^{\text {th }} \mathrm{HCOV}$ that was identified in January 2021 [20]. SARS-CoV-2 belongs to the genus $B$ and showed $98-99 \%$ similarity to bat-derived coronaviruses (bat-SL-CoVZC45 and batSL-CoVZXC21) [21], 50\% to MERS-CoV [22], and $80 \%$ similarity to SARSCoV [23]. This new ß-CoV was named SARS-CoV-2 by the international committee on taxonomy of viruses [24].
Bats and pangolin have been suggested as reservoir of SARS-CoV-2 $[25,26]$. Studies that were conducted by Lai, et al. and Zhou, et al. showed that B-CoV isolated from bats has a sequence similarity of $96 \%$ to $99 \%$ with SARS-CoV-2 [21,26]. Xiao, et al. (2020) demonstrated that CoV from a pangolin and SARS-CoV-2 has very high genetic similarity, with a similarity that ranges from $90-100 \%$ in their structural proteins, suggesting that pangolins can be also the natural host of SARS-CoV-2 [25]. However, a recent study has not substantiated the possible origin of SARS-CoV-2 from pangolin. This was demonstrated by the fact that pangolin virus samples exhibit poor binding to the human ACE2 receptor, which is the primary receptor in the pathogenesis of SARSCoV-2. SARS-CoV-2 has been also shown to infect cats, ferrets and golden hamster $[27,28]$.

SARS-CoV-2 has many variants; which differs in their distribution and transmissibility [29]. The variants of SARS-CoV-2 include: 1) WIV04/2019; 2) Linage B.1.1.207; 3) Lineage B.1.1.7 (also called variant under investigation (VUI-202012/01) or variant of concern 202012/01; 4) Lineage B.1.351 also known as Cluster 5; 501.V2 variant, 5) Lineage P.1 and 6) Lineage B.1.429/CAL.20C/. The sequence WIV04/2019, which is known as sequence zero, is the original sequence infecting humans. The WIV04/2019 was isolated from symptomatic patient on December 2020, which has been used as reference sequence [30]. The VOC202012/01 has the highest transmissibility [31].

As other RNA viruses, SARS-CoV-2 is constantly evolving by random mutations [32], which may explain the basis of the origin of the many SARS-CoV-2 variants since the virus first appeared in December 2020. The mutations in SARS-CoV-2 have been shown to affect the ability of the virus to evade adaptive immune responses from past SARS-CoV-2 infection and vaccination, which may result in an increased risk of reinfection or decreased efficacy of vaccines [33]. In addition, mutations in some SARS-CoV-2 variants have shown reduced susceptibility of the viruses to convalescent plasma and monoclonal antibodies that are being considered for prevention and treatment [34].

\section{Immune response to SARS-CoV-2}

SARS-CoV-2 induces both innate and adaptive immune responses. Cells of innate immune system first recognize the virus by antigen presenting cells (APCs), which will present the antigen of the virus, to CD4+Thelper cells by MHC class 1 that leads to releasing of IL-12 as a co-stimulatory molecule to stimulate the Th1 cell activation [35]. Activation of Th1 cells stimulate CD8-T cells which kills cells that are infected with the virus. CD4 T cells also initiate production of proinflammatory cytokines. IL-17 is a proinflammatory cytokine that increases when SARS-CoV- 2 infection take place, cytokine that recruit monocytes and neutrophils to site of infection and activate other pro-inflammatory 
cytokines such as IL-1, IL-6, IL-8, IL-21 and TNF- $\beta$ [36]. CD4-T-cells also stimulate humoral immune response by inducing production of antigen-specific antibodies via activating $T$ cell-dependent $B$ cells [37]. The antibodies produced are IgM, IgA and IgG in response to SARSCoV-2 infection [38].

Host immune response to the virus may also causes immunopathogenesis. Acute respiratory distress syndrome (ARDS) is the most common immunopathological event for SARS-CoV-2 infection [39]. The major mechanism that causes ARDS is uncontrolled systemic inflammatory response that arises from the release of large amount pro-inflammatory cytokines [40].

SARS-CoV-2 has evolved several mechanisms to escape from host immune response. SARS-CoV-2 infection has been shown to bring about an overall decrease in the transcription of antiviral genes. The virus was demonstrated to lower innate immune response by reducing the production of Type I and III interferons [41]. In addition, it has been shown that SARS-CoV-2 can decrease adaptive immune response. The decrease in adaptive immune response is because of the fact that the virus brings about reduction in T cells and enhances the exhaustion of effector T cells $[42,43]$. Exhaustion and loss in function of effector $T$ cells arises from increased expression of inhibitory receptors such as programmed cell death protein 1 , and T-cell immunoglobulin and mucin domain-containing protein $3[44,45]$.

\section{Pathogenesis and clinical features of COVID-19}

Entry of SARS-CoV-2 requires two transmembrane proteins in host cell; namely ACE- 2 and transmembrane serine protease 2 (TMPRSS2) [46]. The virus begins its life cycle by binding ACE-2 of the host cell by RBD of the spike protein [10]. The TMPRSS2 facilitates fusion of the virus to host cell by cleaving the $S$ protein into its two subunits [46]. After membrane fusion, the genome of the virus is released into the cytoplasm. Following uncoating, replication of the viral genome and protein synthesis occurs in the cytoplasm of host cell $[47,48]$. Finally, assembly of newly formed viral nucleic acids and proteins into viral particles (virions) occur in endoplasmic reticulum (ER) and Golgi that leave host cell by exocytosis [49].

The respiratory system is the primary system that is mainly infected by the virus. However, other systems may also be affected by SARS-CoV-2, which includes gastrointestinal system, excretory system like kidney and vascular system [50,51]. Invasion of the virus to host cell brings about inflammatory changes such as oedema, necrotic changes, which is associated with proinflammatory cytokines such as IL-6, IL-10, tumor necrosis factor, granulocyte colony stimulating factor and monocyte chemoattractant proteins 1 [45]. These changes are connected with lung injury pathogenesis and intestinal and cardiopulmonary damages [45]. Infection with SARS-CoV-2 also brings about hypoxemia that causes change in intracellular $\mathrm{pH}$, electrolyte changes, and accumulation of lactic acid [52].

Although the virus infects people of all age group, people that are thought to be at a higher risk of developing severe disease when get infected with the virus include older people, and people with underlying comorbidities such as diabetes mellitus, hypertension, cardiorespiratory disorders, chronic liver diseases, renal failure, patients with cancer and those receiving immunosuppressive medication as well as pregnant woman [53].

The incubation period of COVID-19 is 5 days, ranging from 2 to 14 days. The most common symptoms and signs at onset of COVID-19 illness include fever, cough, and fatigue, sputum production, headache, hemoptysis, diarrhea, dyspnea and lymphopenia [53]. Additional abnormal features include RNAaemia, pneumonia, ARDS, acute cardiac injury, and ground-glass opacities. The most common abnormalities seen in chest X-rays and computed tomography (CT) are bilateral peripheral ground-glass opacities, with areas of consolidation developing later in the clinical course of COVID-19 [54].

The severity of the disease ranges from asymptomatic infection to critical illness. On basis of the severity of the disease, the clinical features of COVID-19 can be divided into four levels, namely as asymptomatic or presymptomatic infection; mild; moderate, severe, and critical illness [32]. Asymptomatic or presymptomatic infection: refers to a condition in individuals who test positive for SARS-CoV-2 infection using RT-PCR or other diagnostic methods but who have no symptoms that are consistent with COVID-19. Mild Illness: refers to a condition in individuals who have any of the following various signs and symptoms of COVID-19 (e.g., fever, cough, sore throat, malaise, headache, muscle pain, nausea, vomiting, diarrhea, loss of taste and smell) but who do not have shortness of breath, dyspnea, or abnormal chest imaging. Moderate Illness: Refers to a condition in individuals who show evidence of lower respiratory disease during clinical assessment or imaging and who have oxygen saturation $\left(\mathrm{SpO}_{2}\right) \geq$ $94 \%$ on room air at sea level. Severe Illness: refers to a condition in individuals who have $\mathrm{SpO}_{2}<94 \%$ on room air at sea level, respiratory frequency $>30$ breaths $/ \mathrm{min}$. Critical Illness: Refers to a condition in individuals who have respiratory failure, septic shock, and/or multiple organ dysfunctions.

The elderly and those with comorbid diseases, like hypertension, chronic obstructive lung disease, diabetes, cardiovascular disease, rapidly develop acute respiratory distress, septic shock, metabolic acidosis, and coagulation dysfunction [52]. Though most people with COVID-19 develop mild illness, $14 \%$ develop severe disease that requires hospitalization and oxygen support, 
and $5 \%$ require admission to an intensive care unit [55], with $7.1 \%$ of admitted patients ending in death. It has been demonstrated that the major risk factor for severe COVID-19 is inherited from Neandertals [56], which may explain the higher death rate due to COVID-19 in nonAfrican people.

\section{Epidemiology of COVID-19}

COVID-19 has worldwide distribution [1]. Although all people are susceptible to the disease, people that are more likely to develop severe illness when get infected with the virus include; obese individuals, pregnant woman, older people and individuals with underlying comorbidities such as diabetes, cardiovascular diseases, and chronic respiratory diseases [53,57].

SARS-CoV-2 is highly contiguous virus with reproduction number (Ro) estimated around 3.3, which is higher than the Ro (2.7) of SARS-CoV [58], that can be explained by the fact that the SARS-CoV-2 RBD binds to ACE-2 with higher binding affinity than the SARS-CoV [25]. SARS-CoV-2 is primarily transmitted by droplet transmission route [59]. Transmission of the virus may also occur through fecal-oral route and by contact with contaminated materials [31]. Asymptomatic carriers have been demonstrated to transmit the virus. Airborne transmission of small droplets and particles of SARSCoV-2 to persons further than six feet away can also occur [32].

Currently, the disease has incredibly increased [1] and there is a major difference in the burden of COVID-19 in different continents of the world. Up to April 20, 2021, there were 141,549,845 confirmed cases of COVID-19 and 3,021,397 deaths due to the disease in the world [1]. According to WHO, up to September 13, 2021, COVID-19 has been confirmed in over 224,372,38 individuals worldwide and has resulted in more than 4,625,006 deaths [1].

The rapid rise in the number COVID-19 cases can be explained by three interconnected factors [60], which include: - 1) The high reproduction number (Ro = 3.3) of the virus, (which is greater than that of SARS-CoV: Ro $=2.7$ ); 2) The large number of asymptomatic cases; 3 ) The virus can last on surfaces (plastic and steel) for up to three days.

\section{Interventions against COVID-19}

\section{Diagnosis of COVID-19}

Real time reverse-transcription polymerase chain reaction (RT-PCR) is one of the approaches that have been employed for confirmatory diagnosis of COVID-19 in samples from respiratory secretions and blood. The window period of COVID-19 diagnosis using RT-PCR is up to 5 days after exposure before viral nucleic acids can be detected [32]. Although this approach is gold standard for COVID-19 diagnosis, it has high false negative rate (20\%) [61]. False negative results arise from a mutation that occurs in the part of the virus' genome that is detected by RT-PCR [62]. False negative results are more likely to occur when using RT-PCR that relies on only one genetic target. For this reason, a single negative test result does not exclude the possibility of SARS-CoV-2 infection in people who have a high likelihood of infection based on their exposure history and/or their clinical presentation [63]. In order to reduce the possibility of false negative results that arises from using RT-PCR that relies on only one genetic target, there are many commercial nucleic acid amplification tests that use RT-PCR which rely on multiple targets to detect the virus [64].

The other challenge of using RT-PCR for confirmatory diagnosis of COVID-19 is the discordant shedding of virus from the upper respiratory tract (in sputum) and the lower respiratory tract (in bronchial lavage fluid). As the lower respiratory tract specimens have a higher viral load than the upper respiratory tract sample [65], lower respiratory tract sample has to be tested to establish a diagnosis of COVID-19 if an initial upper respiratory tract sample is negative.

SARS-CoV-2 infection can also be detected by antigen-based testing. Antigen-based diagnostic tests perform best early in the course of symptomatic SARSCoV-2 infection, as the viral load is highest in the early course of the disease [32]. The advantages of antigenbased tests include their low cost and rapid turnaround time. The short time required to carry out antigenbased diagnostic tests makes antigen-based tests a good alternative for rapid testing where preventing transmission is critical [32].

As that of RT-PCR, antigen-based tests have high specificity. But the sensitivity of antigen-based diagnostic tests is lower than RT-PCR. Additional RT$P C R$ is recommended when a person who is strongly suspected of having SARS-CoV- 2 infection has a negative result by antigen-based tests, and when a person who is asymptomatic has a positive result by antigen-based tests [32].

The other technique that is used for the diagnosis of COVID-19 is antibody-based test, which can detect $\operatorname{IgM}, \operatorname{IgA}$ and IgG antibodies against the virus [66]. The development of detectable immunoglobulin $M$ (IgM) and immunoglobulin G (IgG) antibodies to SARSCoV-2 (seroconversion) requires at least 21 days after symptom onset [67]. For this reason, serologic testing is not recommended as the sole basis for diagnosing acute SARS-CoV-2 infection. The other problem in serologic diagnosis of COVID-19 is the false positive test results that may occur due to cross-reactivity from pre-existing antibodies to other coronaviruses [32]. As antigenbased tests and RT-PCR sometimes produce false negative results, serologic tests have been used in some settings as an additional diagnostic test for patients who are strongly suspected to have SARS-CoV-2 infection. The use of antibody-based test in combination with RT- 
PCR to detect IgG or total antibodies 3 to 4 weeks after symptom onset maximizes the sensitivity and specificity to detect past infection [32].

In addition to the aforementioned three approaches, chest computed tomography (CT) scan can play an important supplementary role in the diagnosis of COVID-19. As CT has higher sensitivity than RT-PCR [68-70], CT can decrease the chance of false-negative results in the RT-PCR assay if it used as auxiliary method in the diagnosis of COVID-19. The CT scan features of COVID-19 include bilateral ground-glass opacities with crazy-paving pattern and consolidation in the lung periphery [71].

\section{Treatment of COVID-19}

As the pathological findings associated with SARSCoV-2 infection arises from replication of SARS-CoV-2 and dysregulated immune/inflammatory response to SARS-CoV-2 in the early and later stage of COVID-19, respectively [32], it is expected that antiviral therapies would have the greatest effect early in the course of the disease, whereas immunosuppressive/antiinflammatory therapies are likely to be more beneficial in the later stages of COVID-19.

Remdesivir is the only an antiviral drug which has been approved by the United States food and drug administration (FDA) to treat the disease in adults and children who are older than 12 years [72]. The drug blocks RNA synthesis of the virus by blocking RNA-dependent RNA-polymerase [73]. Remdesivir is recommended for use in hospitalized patients who require minimal supplemental oxygen. However, this drug is not recommended for patients who require mechanical ventilation due to the lack of data showing benefit of the drug in patients that require mechanical ventilation [74-76].

The anti-inflammatory drug dexamethasone has been shown to improve survival in hospitalized patients who require supplemental oxygen, with the greatest benefit observed in patients who require mechanical ventilation. Dexamethasone reduces deaths by about $30 \%$ for people on ventilators and by about $20 \%$ for people who needed supplemental oxygen [72]. In addition, dexamethasone has been demonstrated to have better therapeutic effect when it was administered together with Tocilizumab (a monoclonal antibody) [77].

There are also other corticosteroids, such as prednisone, methylprednisolone or hydrocortisone, that have been shown to be effective in improving survival of sever COVID-19 patients when dexamethasone is not available [72]. Therefore, dexamethasone and other corticosteroids are strongly recommended for treatment of severe COVID-19 patients that require supplemental oxygen and mechanical ventilation [7880].
The other type of therapy that has been recommended by FDA to treat COVID-19 is convalescent plasma therapy [81]. Polyclonal antibodies from patients that recovered from COVID-19 have been used for treatment of COVID-19, which showed positive results with rapid recovery [7].

The other strategy that has been employed for the treatment of COVID-19 is monoclonal antibody-based therapies. Bamlanivimab, Etesevimab, imdevimab and Casirivimab are neutralizing monoclonal antibodies that targets and binds different epitopes of RBD of the Spike protein of SARS-CoV-2 [32]. These monoclonal antibodies are developed as combination therapy, namely bamlanivimab in combination with etesevimab and casirivimab in combination with imdevimab. The FDA recommends bamlanivimab in combination with etesevimab and casirivimab in combination with imdevimab for the treatment of mild to moderate COVID-19 patients who are at high risk for progressing to severe disease. However, recent reports have indicated as SARS-COV-2 variants have already developed resistance for bamlanivimab [82]. For this reason, bamlanivimab is not currently recommended for the treatment of COVID-19.

There has been no therapy that has been demonstrated to be effective in treatment of outpatients with mild to moderate COVID-19 who are not at high risk for disease progression [32]. WHO recommends mechanical ventilation for COVID-19 patients with respiratory distress which fails to respond to standard oxygen therapy [81].

\section{Preventive measures for COVID-19}

Interventions that have been employed to prevent SARS-CoV-2 infection include: staying at home, social and physical distancing, quarantine, washing hand with sanitizer, using face mask, immunization, timely diagnosis and treatment of the disease [83].

Effective SARS-CoV-2 vaccines are essential to prevent SARS-CoV-2 infection and control COVID-19 pandemic. There are several vaccine development strategies that have been designed to develop SARS-CoV-2 vaccines, including; a live-attenuated virus, viral vector vaccines, recombinant protein vaccine, inactivated virus vaccines, DNA and RNA-based vaccines. The European Medicines agency and the US FDA set a cutoff of $50 \%$ as the efficacy required to approve a COVID-19 vaccine for use $[84,85]$.

The following are vaccines that have been proven to be effective and safe against SARS-CoV-2 infections [86]:-Comirnaty (mRNA-based vaccine); Moderna COVID-19 vaccine also called mRNA-1273 (mRNA-based vaccine); CoronaVac (inactivated vaccine); COVID-19 Vaccine AstraZeneca (inactivated virus vaccine); Sputnik $V$ (inactivated virus vaccines); BBIBP-CorV (inactivated vaccine); EpiVacCorona (peptide vaccine) and Covaxin (inactivated vaccine) [86]. 
There are also other vaccine candidates that have shown promising result and have reached phase-two and phase three clinical trials [86], which includes JNJ78436735 (non-replicating viral vector); NVX-CoV2373 (Nanoparticle vaccine); Convidicea (Ad5-nCoV) (adenovirus type 5 vector); INO-4800 (plasmid DNAbased vaccine); VnCoV (mRNA-based vaccine); ZF2001 (Recombinant vaccine); and ZyCoV-D (plasmid DNAbased vaccine).

The vaccines that were authorized and approved for COVID-19 have been shown to have positive outcomes. For example, a vaccine efficacy study that was done between December 2020 and January 2021 in Israel on efficacy of Moderna vaccine showed that Moderna vaccines reduces symptomatic COVID-19 and hospitalization due to COVID-19 by more than 99\% [87]. Similarly, a recent study that was conducted between January 2021 and March 2021 in Israel demonstrated that the Pfizer vaccine reduced asymptomatic COVID-19 infections by $94 \%$ and symptomatic COVID-19 infections by $97 \%[88]$.

Furthermore, both the Moderna and Pfzier/BioNTech mRNA vaccines have been shown to have no serious side effects $[87,88]$. Likewise, a vaccine efficacy study carried out in the United States showed that mRNA vaccines were $80 \%$ protective against asymptomatic infections [89]. Another study that was conducted in England that evaluated the efficacy of another COVID-19 vaccine also reported positive result. This study found that OxfordAstraZeneca COVID-19 vaccine is about $73 \%$ effective in prevention of SARS-CoV-2 infection in people aged 70 and older [90].

Although the COVID-19 vaccines substantially reduce the infection rate of SARS-CoV-2, it is still possible for fully vaccinated people to contract and spread SARSCoV-2 [91]. Hence, vaccinated people have to continue using the other preventive measures, such as washing hand with sanitizer, using face mask, social and physical distancing.

The efficacy of COVID-19 can be reduced by the high mutation rate of SARS-CoV-2 [92]. A study showed that many vaccines developed for the initial strain of SARS-CoV-2 have lower efficacy for some variants against symptomatic COVID-19 [93]. For this reason, modification of the current vaccines is required to develop effective vaccines against all variants of the virus.

One of the major challenges of COVID-19 vaccination is ensuring that enough doses are available globally. Scarcity in supply coupled with the large volumes of pre-orders made by richer countries creates challenges to achieving timely, universal access [94,95]. Billions of individuals around the world might not have access to COVID-19 vaccines in 2021 that could prolong the pandemic and raise the risk of further mutations of the virus emerging, which may undermine the efficacy of existing vaccines [96]. In order to avoid uneven access to vaccines in April, 2020, WHO announced the creation of a global allocation mechanism, the COVID-19 Vaccine Global Access (COVAX) Facility, coordinated jointly with CEPI and GAVI. COVAX is a pooled procurement initiative that, in addition to seeking to secure low prices, aims to provide all countries with access to sufficient amount vaccines during the acute phase of the pandemic in 2021 [97]. Self-financing countries can purchase vaccines from COVAX at average price of \$11 per dose, whereas low-income and middle-income countries can buy the vaccines at considerably lower prices (\$1.6$2 \cdot 0$ per dose), subsidized through official development assistance [98].

Face mask is one of the non-pharmaceutical public health measures that have been employed to prevent the spread of SARS-CoV-2. Evidences suggest that face mask wearing reduces transmissibility per contact by reducing transmission of infected respiratory particles in both laboratory and clinical contexts [98]. Public mask wearing is most effective at reducing spread of the virus when compliance is high $[99,100]$. In response the scarcity of medical mask, the adoption of public cloth mask wearing has been recommended as an effective form of source control, in combination with existing hygiene, distancing, and contact tracing strategies [98].

Physical distancing is also a kind of nonpharmaceutical public health measure that has been employed to combat COVID-19 pandemic. Physical distancing of at least $1 \mathrm{~m}$ seems to be strongly associated with a large protective effect, and distances of $2 \mathrm{~m}$ was shown be more effective [101].

The other measure that can be used to reduce the transmission probability of SARS-CoV-2 is contact tracing. It involves identifying individuals who have been in contact with people who are infected with SARSCoV-2 and can be used to find a source of infection by identifying settings or events where infection may have occurred, allowing for targeted public health and social measures [102]. Contact tracing together with robust testing, isolation and care of cases is a key strategy for interrupting chains of transmission of SARS-CoV-2 and reducing COVID-19-associated mortality. The main challenge of contact tracing is the fact that it requires a large resource cost, as it requires trained personnel to perform the tracing. Poor countries may not be able to mobilize a contact tracing task force [102].

\section{Conclusions}

The preventive and control measures that have been employed against COVID-19 include: Staying at home, social and physical distancing, quarantine, washing hand with sanitizer, using face mask, immunization, timely diagnosis and treatment of the disease. The methods that have been used for diagnosis of COVID-19 include: 
Real time-PCR, antigen testing, serology and CT. Real time-PCR is the gold standard for diagnosis of COVID-19. Antiviral drugs that have been approved for treatment of COVID-19 include remedsvier, monoclonal antibodies and convalescent plasma therapy. The vaccines that were authorized and approved for COVID-19 have been shown to have positive outcomes. However, it is still possible for fully vaccinated people to contract and spread SARS-CoV-2 due to the high mutation rate of SARS-CoV-2. As SARS-CoV-2 is constantly changing by random mutation, modification of the current vaccines is required to develop effective vaccines against all variants of the virus. In addition, as the available COVID-19 diagnosis methods have considerable limitations, a novel diagnostic approach that may overcome the limitations has to be designed.

\section{Acknowledgements}

I acknowledge the reviewers of this journal (International Journal of Virology and AIDS) for their constructive comments.

\section{References}

1. WHO (2021) World health organization. Coronavirus (COVID-19) Dashboard.

2. Guo Y, Cao Q, Hong Z, Tan Y, Chen S, et al. (2020) The origin, transmission and clinical therapies on coronavirus disease 2019 (COVID-19) outbreak an update on the status. Mil Med Res 7: 1-10

3. Li Q, Guan X, Wu P, Wang X, Zhou L, et al. (2020) Early transmission dynamics in Wuhan, China, of novel coronavirus infected pneumonia. N Engl J Med 382: 11991207.

4. Graham R, Donaldson E, Baric R (2013) A decade after SARS: Strategies for controlling emerging coronaviruses. Nat Rev Microbiol 11: 836-848.

5. Neuman BW, Adair BD, Yoshioka C, Quispe JD, Orca G, et al. (2006) Supramolecular architecture of severe acute respiratory syndrome coronavirus revealed by electron cryomicroscopy. J Virol 80: 7918-7928.

6. Fehr AR, Perlman S (2015) Coronaviruses: An overview of their replication and pathogenesis. Methods Mol Biol 1282: $1-23$.

7. Jiang S, Hillyer C, Du L (2020) Neutralizing antibodies against SARS-CoV-2 and other human Coronaviruses. Trend Immunol 41: 355-359.

8. Astuti I, Ysrafil (2020) Severe Acute Respiratory Syndrome Coronavirus 2 (SARS-CoV-2): An overview of viral structure and host response. DMS: CRR 14: 407-412.

9. Xu H, Zhong L, Deng J, Peng J, Dan H, et al. (2020) High expression of ACE2 receptor of 2019-nCoV on the epithelial cells of oral mucosa. Int J Oral Sci 12: 1-5.

10. Walls A, Park Y, Tortorici M, Wall A, McGuire A, et al (2020) Structure, function, and antigenicity of the SARSCoV-2 spike glycoprotein. Cell 181: 281-292.

11. Schoeman D, Fielding BC (2019) Coronavirus envelope protein: Current knowledge. Virol J 16: 69

12. Nieto-Torres J, Dediego M, Verdia-Baguena C, JimenezGuardeno J, Regla-Nava J, et al. (2014) Severe acute respiratory syndrome coronavirus envelope protein ion channel activity promotes virus fitness and pathogenesis. PLos Pathog 10: e1004077.

13. Wang M, Ludwig K, Böttcher C, Veit M (2016) The role of stearate attachment to the hemagglutinin-esterase-fusion glycoprotein HEF of influenza C virus. Cell Microb 18: 692704.

14. Cornelissen L, Wierda C, van der Meer F, Herrewegh A, Horzinek M, et al. (1997) Hemagglutinin-esterase, a novel structural protein of torovirus. J Virol 71: 5277-5286.

15. Cui J, Li F, Shi Z (2019) Origin and evolution of pathogenic coronaviruses. Nat Rev Microbiol 17: 181-192.

16. Mclntosh K (2020) Coronaviruses.

17. Tabibzadeh A, Esghaei M, Soltani S, Yousefi $P$, Taherizadeh M, et al. (2021) Evolutionary study of COVID-19, severe acute respiratory syndrome coronavirus 2 (SARS-CoV-2) as an emerging coronavirus: Phylogenetic analysis and literature review. Vet Med Sci 7: 559-571.

18. Weiss S, Navas-Martin S (2005) Coronavirus pathogenesis and the emerging pathogen severe acute respiratory syndrome coronavirus. MMBR 69: 635-664.

19. Rabi F, Zoubi M, Kasasbeh G, Salameh D, Al-Nasser A (2020) SARS-CoV-2 and Coronavirus Disease 2019: What we know so far. Pathogens 9: 231.

20. Zhou P, Fan H, Lan T, Yang X, Shi W, et al. (2018) Fatal swine acute diarrhoea syndrome caused by an HKU2related coronavirus of bat origin. Nature 556: 255-258.

21. Lai C, Shih T, Ko W, Tang H, Hsueh P (2020) Severe acute respiratory syndrome coronavirus 2 (SARS-CoV-2) and coronavirus disease-2019 (COVID-19): The epidemic and the challenges. Int J Antimicrob Agents 55: 105924.

22. Ren L, Wang Y, Wu Z, Xiang Z, Guo L, et al. (2020) Identification of a novel coronavirus causing severe pneumonia in human: a descriptive study. Chinese Med J 133: $1015-1024$.

23. Zimmer K (2021) The scientist magazine ${ }^{\circledR}$. Side-by-side comparisons of important SARS-CoV-2 variants. The Scientist, exploring life, inspiring innovation.

24. WHO (2020) Naming the coronavirus disease (COVID-19) and the virus that causes it.

25. Xiao K, Zhai J, Feng Y, Zhou N, Zhang X, et al. (2020) Isolation of SARS-CoV-2-related coronavirus from Malayan pangolins. Nature 583: 286-289.

26. Zhou P, Yang XL, Wang XG, Hu B, Zhang L, et al. (2020) A pneumonia outbreak associated with a new coronavirus of probable bat origin. Nature 579: 270-273.

27. Sia SF, Yan LM, Chin AWH, Fung K, Choy KT, et al. (2020) Pathogenesis and transmission of SARS-CoV-2 in golden hamsters. Nature 583: 834-838.

28. Shi J, Wen Z, Zhong G, Yang H, Wang C, et al. (2020) Susceptibility of ferrets, cats, dogs, and other domesticated animals to SARS-coronavirus 2. Science 368: 1016-1020.

29. Nguyen T, Pham T, Van T, Nguyen T, Nguyen D, et al. (2020) Genetic diversity of SARS-CoV2and clinical, epidemiological characteristics of COVID-19 patients in Hanoi, Vietnam. PLos One 15: 1-15.

30. GISAID (2021) Official hCoV-19 Reference Sequence.

31. WHO (2020) There is a current outbreak for coronavirus (COVID-19) disease. 
32. NIH (2021) COVID-19 Treatment Guidelines.

33. Walensky RP, Walke HT, Fauci AS (2021) SARS-CoV-2 variants of concern in the United States-challenges and opportunities. JAMA 325: 1037-1038.

34. Wang P, Nair MS, Liu L, Iketani S, Luo Y, et al. (2021) Antibody resistance of SARS-CoV-2 variants $B .1 .351$ and B.1.1.7. Nature 593: 130-135.

35. Shah VK, Firmal P, Alam A, Ganguly D, Chattopadhyay S (2020) Overview of Immune Response During SARSCoV-2 Infection: Lessons from the Past. Front Immunol 11: 1949.

36. Zumla A, Hui D, Azhar E, Memish Z, Maeurer M (2020) Reducing mortality from 2019-nCoV: Host-directed therapies should be an option. Lancet 395: e35-e36.

37. Li X, Geng M, Peng Y, Meng L, Lu S (2020) Molecular immune pathogenesis and diagnosis of COVID-19. J Pharm Anal 10: 1-17.

38. Schroeder $H$, Cavacini $L$ (2010) Structure and Function of Immunoglobulins. J Allergy Clin Immunol 125: S41-S52.

39. Xu Z, Shi L, Wang Y, Zhang J, Huang L, et al. (2020) Pathological findings of COVID19 associated with acute respiratory distress syndrome. Lancet Resp Med 8: 420422

40. Cameron MJ, Bermejo-Martin J, Danesh A, Muller MP, Kelvin DJ (2008) Human immunopathogenesis of severe acute respiratory syndrome (SARS). Virus Res 133: 13-19.

41. Blanco-Melo D, Nilsson-Payant BE, Liu W, Uhl S, Hoagland D, et al. (2020) Imbalanced host response to SARS-CoV-2 drives development of COVID-19. Cell 181: 1036-1045.

42. Diao B, Wang C, Tan Y, Chen X, Liu Y, et al. (2020) Reduction and functional exhaustion of $T$ cells in patients with coronavirus disease 2019 (COVID-19). Front Immunol 11: 1-14.

43. Zheng $\mathrm{H}$, Zhang M, Yang C-X, Zhang N, Wang X-C, et al. (2020) Elevated exhaustion levels and reduced functional diversity of $\mathrm{T}$ cells in peripheral blood may predict severe progression in COVID-19 patients. Cell Mol Immunol 17: 541-543.

44. Qin C, Zhou L, Hu Z, Zhang S, Yang S, et al. (2020) Dysregulation of immune response in patients with COVID-19 in Wuhan, China. Clin Infect Dis 71: 762-768.

45. Chiappelli A, Kashakshooy A, Greenberg G (2020) COVID-19 immunopathology and immunotherapy. Bioinformation 16: 219-222.

46. Hoffmann M, Weber H, Schroeder S, Kruger N, Herrler T, et al. (2020) SARSCoV-2 Cell Entry Depends on ACE2 and TMPRSS2 and Is Blocked by a Clinically Proven Protease Inhibitor. Cell 181: 271-280.

47. Hussain S, Pan J, Chen Y, Yang Y, Xu J, et al. (2005) Identification of novel subgenomic RNAs and noncanonical transcription initiation signals of severe acute respiratory syndrome coronavirus. J Virol 79: 5288-5295.

48. Pascual MR (2020) Coronavirus SARS-CoV-2: Analysis of subgenomic mRNA transcription, 3CLpro and PL2pro protease cleavage sites and protein synthesis.

49. Perrier A, Bonnin A, Desmarets L, Danneels A, Goffard A, et al. (2019) The C-terminal domain of the MERS coronavirus $\mathrm{M}$ protein contains a trans-Golgi network localization signal. J Biol Chem 294: 14406-14421.

50. Bansal M (2020) Cardiovascular disease and COVID-19. Diabetes Metab Syndr 14: 247-250.
51. Liu PP, Blet A, Smyth D, Li H (2020) The science underlying COVID-19: Implications for the cardiovascular system. Circulation 142: 68-78.

52. Azer SA (2020) COVID-19: Pathophysiology, diagnosis, complications and investigational therapeutics. New Microbe and New Infect 37: 100738.

53. Wang W, Xu Y, Gao R, Lu R, Han K, et al. (2020) Detection of SARS-CoV-2 in different types of clinical specimens. JAMA 323: 1843-1844.

54. Shi H, Han X, Jiang N, Cao Y, Alwalid O, et al. (2020) Radiological findings from 81 patients with COVID-19 pneumonia in Wuhan, China: A descriptive study. Lancet Infect Dis 20: 425-434.

55. WHO (2020) Clinical management of severe acute respiratory infection (SARI) when COVID19 disease is suspected: Interim guidance.

56. Zeberg H, Pääbo S (2020) The major genetic risk factor for severe COVID-19 is inherited from Neandertals. Nature 587: 610-622.

57. CDC (2020) Centers for Disease Control and Prevention. Evidence used to update the list of underlying medical conditions that increase a person's risk of severe illness from COVID-19.

58. Riley S, Fraser C, Donnelly C, Ghani A, Abu-Raddad L, et al. (2003) Transmission dynamics of the etiological agent of SARS in Hong Kong: Impact of public health interventions. Science 300: 1961-1966.

59. Rothan A, Byrareddy N (2020) The epidemiology and pathogenesis of coronavirus disease (COVID-19) outbreak. J Auto Immun 109: 102433.

60. OECD (2020) OECD Policy Responses to Coronavirus (COVID-19). Flattening the COVID-19 peak: Containment and mitigation policies. Tackling Coronavirus (COVID-19). Contributing to a global effort.

61. Xiao AT, Tong YX, Zhang S (2020) False-negative of RTPCR and prolonged nucleic acid conversion in COVID-19: Rather than recurrence. J Med Virol 92: 1755-1756.

62. FDA. Food and Drug Administration (2021) Genetic variants of SARS-CoV-2 may lead to false negative results with molecular tests for detection of SARS-CoV-2-letter to clinical laboratory staff and health care providers.

63. Kucirka LM, Lauer SA, Laeyendecker O, Boon D, Lessler $J$ (2020) Variation in false-negative rate of reverse transcriptase polymerase chain reaction-based SARSCoV-2 tests by time since exposure. Ann Intern Med 173: 262-267.

64. CDC (2021) Centers for Disease Control and Prevention. Science brief: Emerging SARS-CoV-2 variants.

65. Hase R, Kurita T, Muranaka E, Sasazawa $H$, Mito $H$, et al. (2020) A case of imported COVID-19 diagnosed by PCRpositive lower respiratory specimen but with PCR-negative throat swabs. Infect Dis (Lond) 52: 423-426.

66. Loeffelholz J, Tang W (2020) Laboratory diagnosis of emerging human coronavirus infections- the state of the art. Emerg Microbe Infect 9: 747-756.

67. Okba N, Muller M, Li W, Wang C, Geurtsvan Kessel C, et al. (2020) Severe acute respiratory syndrome coronavirus 2-specific antibody responses in coronavirus disease patients. Emerg Infect Dis 26: 1478-1488.

68. Ai T, Yang Z, Hou H, Zhan C, Chen C, et al. (2020) Correlation of chest CT and RT-PCR testing in coronavirus 
disease 2019 (COVID-19) in China: A report of 1014 cases. Radiology 296: E32-E40.

69. Fang $\mathrm{Y}$, Zhang $\mathrm{H}$, Xie J, Lin M, Ying L, et al. (2020) Sensitivity of chest CT for COVID-19: Comparison to RTPCR. Radiology 296: E115-E117.

70. Xie X, Zhong Z, Zhao W, Zheng C, Wang F, et al. (2020) Chest CT for typical 2019-nCoV pneumonia: relationship to negative RT-PCR testing. Radiology 296: E41-E45.

71. Kanne JP (2020) Chest CT findings in 2019 nove coronavirus (2019-nCoV) infections from Wuhan, China: Key points for the radiologist. Radiology 295: 16-17.

72. COVID-19 (coronavirus) drugs: Are there any that work?

73. CTG (2021) COVID-19 Treatment Guidelines. Remdesivir.

74. Beigel JH, Tomashek KM, Dodd LE, Dodd L, Mehta A, et al. (2020) Remdesivir for the treatment of COVID-19-final report. N Engl J Med 383: 1813-1826.

75. Goldman J, Lye D, Hui D, Marks K, Bruno R, et al. (2020) Remdesivir for 5 or 10 days in patients with severe COVID-19. N Engl J Med 383: 1827-1837.

76. Spinner CD, Gottlieb RL, Criner GJ, Criner G, Lopez J, et al. (2020) Effect of remdesivir vs standard care on clinical status at 11 days in patients with moderate COVID-19: A randomized clinical trial. JAMA 324: 1048-1057.

77. Gordon AC, Mouncey PR, Al-Beidh F, Rowan K, Nichol K et al. (2021) Interleukin-6 receptor antagonists in critically ill patients with COVID-19. N Engl J Med 384: 1491-1502.

78. Angus DC, Derde L, Al-Beidh F, Annane A, Arabi $\mathrm{Y}$, et al. (2020) Effect of hydrocortisone on mortality and organ support in patients with severe COVID-19: The REMAPCAP COVID-19 corticosteroid domain randomized clinical trial. JAMA 324: 1317-1329.

79. Jeronimo C, Farias M, Val F, Sampaio, V, Alexandre M, et al. (2020) Methylprednisolone as adjunctive therapy for patients hospitalized with COVID-19 (Metcovid): A randomised, double-blind, Phase Ilb, placebo-controlled trial. Clin Infect Dis 72: e373-e381.

80. Tomazini BM, Maia IS, Cavalcanti AB, Berwanger O, Rosa $R$, et al. (2020) Effect of dexamethasone on days alive and ventilator-free in patients with moderate or severe acute respiratory distress syndrome and COVID-19: The CoDEX randomized clinical trial. JAMA 324: 1307-1316.

81. WHO (2020) Estimating mortality from COVID-19.

82. CDC (2021) Centers for Disease Control and Prevention. SARS-CoV-2 variant classifications and definitions.

83. CDC (2021) Centers for disease control and prevention. COVID-19. How to Protect Yourself \& Others.

84. EMA (2021) EMA sets $50 \%$ efficacy goal - with flexibility -for COVID vaccines".

85. FDA (2021) The FDA's cutoff for Covid-19 vaccine effectiveness is 50 percent. What does that mean?".
86. Craven J (2021) COVID-19 vaccine tracker/regulatory affairs professional society/RAP.

87. Pfizer (2021) Real-world evidence confirms high effectiveness of Pfizer-BioNTech COVID-19 vaccine and profound public health impact of vaccination one year after pandemic declared.

88. Amir T, Elizabeth C (2021) Israel's health data suggests Pfizer and Moderna vaccines may be more effective than we thought. CNN.

89. Tande A, Pollock B, Shah N, Farrugia G, Virk A, et al. (2021) Impact of the COVID-19 vaccine on asymptomatic infection among patients undergoing pre-procedural COVID-19 molecular screening. Clin Infect Dis.

90. Bernal JL, Andrews N, Gower C, Robertson C, Stowe J, et al. (2021) Effectiveness of the Pfizer-BioNTech and OxfordAstraZeneca vaccines on COVID-19 related symptoms, hospital admissions, and mortality in older adults in England: test negative case-control study. BMJ 373: n1088.

91. Goodman P, Mandavilli A, Robbins R, Stevis-Gridneff M (2021) What would it take to vaccinate the world against COVID? The New York times.

92. FDA (2021) Coronavirus (COVID-19) Update: FDA Issues Policies to Guide Medical Product Developers Addressing Virus Variants".

93. Mahase E (2021) Covid-19: Where are we on vaccines and variants? BMJ 372: n597.

94. CEPI (2020) Coalition for epidemic preparedness innovations. Manufacturing survey results analysis.

95. Khamsi $R$ (2020) If a coronavirus vaccine arrives, can the world make enough? Nature 580: 578-580.

96. Wouters OJ, Shadlen KC, Salcher-Konrad M, Pollard AJ, Heidi L, et al. (2021) Challenges in ensuring global access to COVID-19 vaccines: Production, affordability, allocation, and deployment. Lancet 397: 1023-1034.

97. GAVI (2020) The Vaccine Alliance. COVAX explained.

98. Howarda J, Huangc A, Tufekcie Z, Zdimalf V, Westhuizeng $\mathrm{H}$, et al. (2021) An evidence review of face masks against COVID-19. PNAS 118: e2014564118.

99. Gupta M, Gupta K, Gupta S (2020) The use of facemasks by the general population to prevent transmission of COVID-19 infection: A systematic review (Preprint). MedRxiv.

100. The University of Edinburgh Usher Institute (2020) Summary: Does the use of face masks in the general population make a difference to spread of infection?

101. Chu DK, Akl EA, Duda S, Solo K, Yaacoub S, et al. (2020) Physical distancing, face masks, and eye protection to prevent person-to-person transmission of SARS-CoV-2 and COVID-19: A systematic review and meta-analysis. Lancet 395: 1973-1987.

102. WHO (2021) Contact tracing in the context of COVID-19. World Health Organization. Interim guidance. 